\title{
Clinical and procedural predictors of no-reflow in patients with acute myocardial infarction after primary percutaneous coronary intervention
}

\author{
Hua Zhou, Xiao-yan He, Shao-wei Zhuang, Juan Wang, Yan Lai, Wei-gang Qi, Yi-an Yao, Xue-bo Liu \\ Department of Cardiology, East Hospital, Tongji University School of Medicine, Shanghai 200120, China
}

Corresponding Author: Hua Zhou,Email: zhzju0560@sohu.com

BACKGROUND: The treatment of acute myocardial infarction (AMI) is thought to restore antegrade blood flow in the infarct-related artery (IRA) and minimize ischemic damage to the myocardium as soon as possible. The present study aimed to identify possible clinical predictors for no-reflow in patients with AMI after primary percutaneous coronary intervention (PCI).

METHODS: A total of 312 consecutive patients with AMI who had been treated from January 2008 to December 2010 at the Cardiology Department of East Hospital, Tongji University School of Medicine were enrolled in this study. Inclusion criteria were: (i) patients underwent successfully primary $\mathrm{PCl}$ within 12 hours after the appearance of symptoms; or (ii) patients with ischemic chest pain for more than 12 hours after a successful primary $\mathrm{PCl}$ within 24 hours after appearance of symptoms. Exculsion criteria were: (i) coronary artery spasm; (ii) diameter stenosis of the culprit lesion was $\leq 50 \%$ and coronary blood flow was normal; (iii) patients with severe left main coronary or multivessel disease, who had to require emergency revascularization. According to thrombolysis in myocardial infarction (TIMI), the patients were divided into a reflow group and a no-reflow group. The clinical data, angiography findings and surgical data were compared between the two groups. Univariate and multivariate logistic regressions were used to determine the predictors for no-reflow.

RESULTS: Fifty-four (17.3\%) of the patients developed NR phenomenon after primary $\mathrm{PCl}$. Univariate analysis showed that age, time from onset to reperfusion, systolic blood pressure (SBP) on admission, Killip class of myocardial infarction, intra-aortic balloon pump (IABP) use before primary $\mathrm{PCl}$, TIMI flow grade before primary $\mathrm{PCl}$, type of occlusion, thrombus burden on baseline angiography, target lesion length, reference luminal diameter and method of reperfusion were correlated with no-reflow $(P<0.05$ for all). Multiple logistic regression analysis identified that age $>65$ years [OR=1.470,95\% confidence interval $(\mathrm{Cl}) 1.460-1.490, P=0.007]$, long time from onset to reperfusion $>6$ hours $(O R=1.270,95 \% \mathrm{Cl} 1.160-1.400, P=0.001)$, low SBP on admission $<100 \mathrm{mmHg}$ $(O R=1.910,95 \% \mathrm{Cl} 1.018-3.896, P=0.004)$, IABP use before $\mathrm{PCl}(\mathrm{O} R=1.949,95 \% \mathrm{Cl} 1.168-3.253$, $P=0.011)$, low $(\leq 1)$ TIMI flow grade before primary $\mathrm{PCl}(O R=1.100,95 \% \mathrm{Cl} 1.080-1.250, P<0.001)$, high thrombus burden $(O R=1.600,95 \% \mathrm{Cl} 1.470-2.760, P=0.030)$, and long target lesion $(O R=1.948$, $95 \% \mathrm{Cl} 1.908-1.990, P=0.019)$ on angiography were independent predictors of no-reflow.

CONCLUSION: The occurrence of no-reflow after primary $\mathrm{PCl}$ for acute myocardial infarction can predict clinical, angiographic and procedural features.

KEY WORDS: Acute myocardial infarction; No-reflow phenomenon; Percutaneous coronary intervention; Thrombus

World J Emerg Med 2014;5(2):96-102 DOI: $10.5847 /$ wjem.j.issn.1920-8642.2014.02.003 


\section{INTRODUCTION}

The treatment of acute myocardial infarction (AMI) is thought to restore antegrade blood flow in the infarctrelated artery (IRA) and minimize ischemic damage to the myocardium as soon as possible. Primary percutaneous coronary intervention (PCI) is the most efficient way to restore antegrade blood flow in the current management of ST-elevation AMI. Despite the recent progress in PCI, however, a proportion of patients develop epicardial coronary artery reperfusion but not myocardial reperfusion after primary PCI, known as no-reflow. Patients who have developed no-reflow are at an increased risk for left ventricular dysfunction and progressive myocardial damage. The present study was undertaken to identify clinical factors, angiographic findings and procedural features, which predict the no-reflow phenomenon in patients with AMI after primary PCI.

\section{METHODS Patients}

A total of 312 consecutive AMI patients who had undergone emergency PCI between January 2008 and December 2010 at the Cardiology Department of East Hospital, Tongji University School of Medicine were enrolled in the study. Inclusion criteria were: (i) patients underwent successfully primary PCI within 12 hours after appearance of symptoms; and (ii) patients with ischemic chest pain for more than 12 hours who underwent successfully primary PCI within 24 hours after appearance of symptoms. AMI was defined as typical chest pain for more than 30 minutes and either ST segment elevation of $>1 \mathrm{~mm}$ in 2 consecutive leads or the onset of left bundle-branch block with 2-fold elevation of creatine kinase $(\mathrm{CK})$ and creatine kinase-MB (CK$\mathrm{MB}$ ) fraction. Exclusion criteria were: patients who were treated conservatively for coronary artery spasm or $\leq 50 \%$ diameter stenosis of the culprit lesion with normal coronary blood flow; those who required emergency revascularization for severe left main coronary artery or mutlivessel diseases; those with saphenous vein grafts or left internal mammary artery lesions; and those who did not achieve coronary artery patency.

\section{Coronary angiography and primary $\mathrm{PCI}$}

All patients were subjected to oral aspirin $(300 \mathrm{mg})$ and clopidogrel (300 mg), as well as intravenous 8 000-10 000 $\mathrm{U}$ unfractionated heparin. They recieved PCI through the femoral artery or the radial artery. Before the PCI, standard left and right coronary angiograms with at least 2 best projections were obtained for each patient. Two experienced interventional cardiologists assessed a set of parameters for each angiogram and reached consensus on the findings. These parameters included: morphology of the IRA, Rentrop collateral flow, angiographic features of the target lesion, TIMI flow grades before and after primary PCI, culprit lesion stenosis degree, target lesion length, and luminal diameter. Angiographic data of the lesion responsible for the infarction were recorded: (i) thrombus burden (mild, moderate or high); (ii) types of total occlusion if present (tapered or cutoff lesion); (iii) types of lesion if subtotal occlusion is present (eccentric or concentric lesion); (iv) length of target lesion; and (v) lesion location (proximal, mid or distal lesion). Thrombus burden was scored in five degrees according to Gibson, ${ }^{[1]}$ it was classified as mild if the TIMI thrombus was class 0 and 1 , moderate if the TIMI thrombus was class 2 and 3, and high if the TIMI thrombus was more than class 3 . The reperfusion therapy (balloon angioplasty or stent placement) was determined by physician's discretion during primary PCI. Stent placement was strongly encouraged unless the IRA was heavily calcified or reference luminal diameter (RLD) $<2.25 \mathrm{~mm}$. Drug-eluting stents were used for all stenting procedures. Each patient was treated with clopidogrel for at least 1 year after PCI. Infarct size was estimated by the measurement of peak activity of serum creatine kinase $(\mathrm{CK})$, and peak enzyme release was assessed using 5-6 serial measurements taken at the first 4 days after appearance of symptoms. ST-segment resolution was evaluated before and 1 hour after primary PCI.

All patients were divided into two groups based on the post-procedural TIMI flow in the IRA: TIMI flow $\leq 2$ (no-reflow) and TIMI flow 3 (reflow). The patient was considered to exhibit a no-reflow phenomenon if blood flow in the IRA was a $T I M I \leq 2$ flow despite successful dilatation and absence of mechanical complications such as dissection, spasm or angiographically evident distal embolization after completion of the procedure. ${ }^{[2]}$

\section{Statistical analysis}

All variables were expressed as mean \pm standard deviation. The Chi-square test was used to analyze categorical variables. Student's $t$ test and analysis of variance were used for continuous variables. Univariate and multivariate analyses were performed to identify independent predictors of no-reflow phenomenon. Statistical analysis was made using SPSS 19.0. A $P$ value $<0.05$ was considered statistically significant. 


\section{RESULTS}

\section{Baseline clinical characteristics}

In the 312 patients who had undergone primary PCI, 54 (17.3\%) showed an angiographic no-reflow phenomenon. The baseline clinical characteristics are shown in Table 1. There were no significant differences between the reflow group and the no-reflow group in sex distribution, hypertension, diabetes mellitus, hypercholesterolemia, current smoking, family history of coronary artery disease, primary MI, and infarct localization or pre-infarction angina ( $P>0.05$ for all). Compared with the reflow group, the no-reflow group had a higher mean age $(67.1 \pm 14.9$ vs. $61.1 \pm 12.4$ years for no-reflow and reflow, respectively), a longer mean reperfusion time $(6.7 \pm 3.2$ vs. $5.4 \pm 2.8$ hours, respectively), a lower level of SBP in admission (101.2 \pm 25.8 vs. $116.2 \pm 22.4$, respectively), a higher level of CK (239 \pm 205 vs. $160 \pm 166 \mathrm{U} / \mathrm{L}$, respectively) $(P<0.05$ for all). Moreover, there were significant differences between the reflow and no-reflow groups in respect to Killip classes before PCI and IABP use before PCI ( $P<0.05$ for all).

\section{Angiographic findings and primary $\mathrm{PCl}$ characteristics}

The angiographic data and procedural features

Table 1. Basline clinical data in the reflow group and the no-reflow group $(n, \%)$

\begin{tabular}{|c|c|c|c|c|}
\hline Variables & $\begin{array}{l}\text { Reflow } \\
(n=258)\end{array}$ & $\begin{array}{c}\text { No-reflow } \\
(n=54)\end{array}$ & $t / \chi^{2}$ value & $P$ value \\
\hline Age(yr) & $61.1 \pm 12.4$ & $67.1 \pm 14.9$ & 24.703 & $<0.01$ \\
\hline Male & $160(62.0)$ & $38(70.3)$ & 1.682 & 0.324 \\
\hline Hypertension & $94(36.4)$ & $23(42.5)$ & 1.152 & 0.224 \\
\hline Diabetes mellitus & $41(15.9)$ & $10(18.5)$ & 0.438 & 0.635 \\
\hline Hypercholesterolemia & $121(46.9)$ & $29(53.7)$ & 1.652 & 0.174 \\
\hline Current smoker & $90(34.8)$ & $20(37.0)$ & 1.120 & 0.295 \\
\hline Family history of CAD & $88(34.1)$ & $19(35.2)$ & 0.436 & 0.829 \\
\hline Previous MI & $13(5.0)$ & $3(5.6)$ & 0.024 & 0.660 \\
\hline Pre-infarction angina & $78(30.3)$ & $17(31.4)$ & 0.576 & 0.841 \\
\hline \multicolumn{5}{|l|}{ Infarct loction } \\
\hline Anterior & $157(60.8)$ & $36(66.7)$ & & \\
\hline Inferior & $79(30.6)$ & $14(25.9)$ & 1.832 & 0.494 \\
\hline Other locations & $22(8.6)$ & $4(7.4)$ & & \\
\hline \multicolumn{5}{|c|}{ Physical findings on admission } \\
\hline $\mathrm{SBP}(\mathrm{mmHg})$ & $116.2 \pm 22.4$ & $101.2 \pm 25.8$ & 24.734 & $<0.001$ \\
\hline DBP (mmHg) & $71.4 \pm 13.4$ & $66.1 \pm 16.8$ & 9.973 & 0.050 \\
\hline Peak CK-MB (U/L) & $160 \pm 166$ & $239 \pm 205$ & 11.762 & 0.014 \\
\hline Reperfusion time (h) & $5.4 \pm 2.8$ & $6.7 \pm 3.2$ & 5.678 & 0.011 \\
\hline Killip classes $(\%)$ & & & 9.653 & 0.02 \\
\hline 1 & $176(68.2)$ & $24(44.4)$ & & \\
\hline 2 & $64(24.8)$ & $20(37)$ & & \\
\hline 3 & $13(5.0)$ & $2(3.7)$ & & \\
\hline 4 & $5(2)$ & $8(14.9)$ & & \\
\hline IABP use before PCI (\%) & $20(7.8)$ & $14(25.9)$ & 42.8 & $<0.0001$ \\
\hline
\end{tabular}

Data were presented as mean \pm SD or number (\%) of patients; $C A D$ : coronary artery disease; MI: myocardial infarction; SBP: systolic blood pressure; DBP: diastolic blood pressure; CK: creatine kinase; IABP: intra-aortic balloon pump. revealed that no-reflow was more frequent in patients who had a low $(\leq 1)$ initial TIMI flow $(92.6 \%$ vs. $69.8 \%)$, a total cut-off occlusion $(42.6 \%$ vs. $29.1 \%)$, a long target lesion ( $21.6 \pm 9.25$ vs. $18 \pm 6.48 \mathrm{~mm})$, and a large vessel diameter ( $3.3 \pm 0.4$ vs. $3.0 \pm 0.3 \mathrm{~mm}$, respectively). Moreover, the no-reflow incidence was significantly higher in patients with a delayed reperfusion $(>6$ hours) and a high rhrombus burden. Reperfusion had an influence on the incidence of no-reflow $(P<0.05)$. However, IRA, the presence of multivessel diseases, locations of target lesions, types of lesions in subtotal occlusions, grades of collateral flows, number of implanted stents, maximal inflation pressures, and repeated balloon dilatations did not affect the incidence of no-reflow $(P>0.05)$ (Table 2).

Table 2. Angiographic and surgical data in the reflow group and the no-reflow group $(n, \%)$

\begin{tabular}{|c|c|c|c|c|}
\hline Variables & $\begin{array}{l}\text { Reflow } \\
(n=258)\end{array}$ & $\begin{array}{c}\text { No-reflow } \\
(n=54)\end{array}$ & $t / \chi^{2}$ value & $P$ value \\
\hline Multivessel disease & $138(53.5)$ & $32(59.3)$ & 0.873 & 0.326 \\
\hline IRA & & & 1.743 & 0.568 \\
\hline LAD & $152(58.9)$ & $36(66.7)$ & & \\
\hline LCX & $23(8.9)$ & $4(7.4)$ & & \\
\hline RCA & $83(32.1)$ & $14(25.9)$ & & \\
\hline Initial TIMI flow & & & 32.742 & 0.0007 \\
\hline $0 / 1$ & $180(69.8)$ & $50(92.6)$ & & \\
\hline $2 / 3$ & $78(30.2)$ & $4(7.4)$ & & \\
\hline Target lesion location & & & 1.172 & 0.386 \\
\hline Proximal & $93(36.0)$ & $21(38.9)$ & & \\
\hline Mid & $159(61.6)$ & $30(55.6)$ & & \\
\hline Distal & $6(2.4)$ & $3(5.5)$ & & \\
\hline \multicolumn{5}{|l|}{ Type of occlusion } \\
\hline Subtotal & 103(39.9) & $11(20.3)$ & & \\
\hline Tapered & $80(31.0)$ & $20(37.1)$ & 9.763 & 0.024 \\
\hline Cut-off & $75(29.1)$ & $23(42.6)$ & & \\
\hline \multicolumn{3}{|c|}{ Lesion types in subtotal occlusion } & 1.362 & 0.428 \\
\hline Eccentric & $23(22.3)$ & $4(36.4)$ & & \\
\hline Concentric & $80(77.7)$ & $7(63.7)$ & & \\
\hline Target lesion length (mm) & $18 \pm 6.48$ & $21.6 \pm 9.25$ & 8.324 & $<0.0001$ \\
\hline Reference diameter (mm) & $3.1 \pm 0.3$ & $3.3 \pm 0.4$ & 2.213 & 0.029 \\
\hline Thrombus burden & & & 23.762 & 0.010 \\
\hline Low & $70(27.1)$ & $9(16.7)$ & & \\
\hline Moderate & $75(29)$ & $12(22.2)$ & & \\
\hline High & $113(43.9)$ & $33(61.1)$ & & \\
\hline Good collateral flow & $16(6.2)$ & $4(7.4)$ & 0.532 & 0.635 \\
\hline Method of reperfusion & & & 7.634 & 0.002 \\
\hline Balloon angioplasty & $9(3.4)$ & $4(7.4)$ & & \\
\hline Stenting after pre-dilation & $163(63.2)$ & $44(81.4)$ & & \\
\hline Direct stenting & $86(33.4)$ & $6(11.2)$ & & \\
\hline $\begin{array}{l}\text { Repeated balloon } \\
\text { dilatations }(\geq 2)\end{array}$ & $79(30.6)$ & $23(42.6)$ & 2.384 & 0.054 \\
\hline Multiple stents $(\geq 2)$ & $43(16.7)$ & $15(27.8)$ & 2.765 & 0.051 \\
\hline Aspiration thrombetomy & $164(63.5)$ & $32(59.3)$ & 0.876 & 0.628 \\
\hline Tirofiban use & $180(69.8)$ & $40(74)$ & 0.435 & 0.644 \\
\hline
\end{tabular}

Data were presented as mean \pm SD or the number of patients; IRA: infarct-related artery; LAD: left anterior descending artery; LCA: left circumfles artery; RCA: right coronary artery; TIMI: thrombolysis in myocardial infarction. 


\section{Independent predictors of no-reflow phenomenon}

Univariate and multivariate analyses identified that age $>65$ years $(O R=1.470,95 \%$ CI $1.460-1.490$, $P=0.007)$, reperfusion time $>6$ hours $(O R=1.270,95 \% \mathrm{CI}$ $1.160-1.400, P=0.001)$, SBP on admission $\leq 100 \mathrm{mmHg}$ $(O R=1.910,95 \%$ CI $1.018-3.896, P=0.004)$, IABP use before PCI ( $O R=1.949,95 \% \mathrm{CI} 1.168-3.253, P=0.011)$, a low initial TIMI flow $(\leq 1)(O R=1.100,95 \%$ CI 1.080 $1.250, P<0.001)$, a high thrombus burden $(O R=1.600$, $95 \%$ CI $1.470-2.760, P=0.030$ ), and a long target lesion $(O R=1.948,95 \%$ CI $1.908-1.990, P=0.019)$ were the independent predictors of the no-flow phenomenon (Table 3).

\section{DISCUSSION}

The rate of no-reflow phenomenon after primary PCI in our study (17.3\%) was similar to that (5\%-25\%) reported previously. ${ }^{[3]}$ The cause of no-reflow after primary PCI in patients with STEMI is complex. The possible mechanisms of no-reflow include endotheliar dysfunction, microvascular disordes, spasm, embolization, and reperfusion injury. Advanced age, delayed reperfusion, a low TIMI flow to PCI, SBP on admission $<100 \mathrm{mmHg}$, intra-aortic balloon pump (IABP) use before PCI, a long target lesion and a high thrombus burden were independent predictors of noreflow. Wang et $\mathrm{al}^{[4]}$ showed that on admission plasma glucose $>13 \mathrm{mmol} / \mathrm{L}$ was one of the no-reflow predictors.

In-hospital and long-term mortality rates are higher in elderly patients with AMI. The success rate of primary PCI in these patients is lower than in younger patients because of delayed hospitalization and increased co-morbidities. Diffuse coronary atherosclerosis, severe vascular calcification, distal microembolization and microcirculation dysfunction are more common in the elderly patients. These pathological changes are related to advanced age, and absence of ischemic preconditioning and collateral circulation, and altered neurohormonal and autonomic influences. They may contribute to distal embolization during primary PCI, resulting in no-reflow. ${ }^{[5,6]}$

Delayed reperfusion (a long duration from onset to reperfusion) is related to no-reflow. Our study demonstrates that patients with a long duration of reperfusion ( $>6$ hours) had a significantly greater thrombus burden and a 1.3-fold increase in no-reflow rate than patients with a short duration of reperfusion. Myocardial necrosis occurs in about 6 hours after the appearance of coronary occlusion. As reported, prolonged ischemia leads to edema of distal capillary beds, swelling of myocardial cells, neutrophil plugging, alterations of capillary integrity, and disruption of microvascular bed, ${ }^{[7]}$ which contribute to the pathogensis of no-reflow. ${ }^{[8]}$ In the early stages of AMI, the thrombus is rich in thrombocytes and relatively easier to lyse adjunctive pharmacrotherapy. With a longer duration to reperfusion, the thrombus takes on more erythrocytes and becomes more rigid. Such thrombi tend to fragment with balloon dilatation, which can lead to distal coronary embolization. Furthermore, delayed reperfusion results in an older well-organized intracoronary thrombus. This may increase the risk of distal embolization during primary $\mathrm{PCI}$ and reduce the likelihood of achieving TIMI 3 flow after the procedure. ${ }^{[9]}$ Yip et al ${ }^{[10]}$ demonstrated that in patients with AMI who had a high thrombus burden, the rate of no-reflow was lower than in those with reperfusion less than 4 hours. This indicates the possible correlation of a thrombus burden with the duration of reperfusion. In case of a long duration of reperfusion and a high thrombus burden, the use of a distal protection device

Table 3. Independent predictors of no-reflow phenomenon shown by univariate and multivariate analyses

\begin{tabular}{|c|c|c|c|c|}
\hline \multirow{2}{*}{ Variables } & \multicolumn{2}{|c|}{ Univariate analysis } & \multicolumn{2}{|c|}{ Multivariate analysis } \\
\hline & $P$ value & $O R(95 \% \mathrm{CI})$ & $P$ value & $O R(95 \% \mathrm{CI})$ \\
\hline Age $(>65$ yr $)$ & $<0.0001$ & $1.884(1.830-1.980)$ & 0.007 & $1.470(1.460-1.490)$ \\
\hline Reperfusion time ( $>6$ hours) & 0.005 & $1.564(1.362-1.872)$ & 0.001 & $1.270(1.16-1.40)$ \\
\hline $\mathrm{SBP}$ on admission $<100 \mathrm{mmHg}$ & 0.005 & $1.570(1.390-1.850)$ & 0.004 & $1.910(1.018-3.896)$ \\
\hline IABP use before PCI & 0.003 & $1.879(1.152-3.172)$ & 0.011 & $1.949(1.160-3.253)$ \\
\hline Initial TIMI flow (0-1) & 0.002 & $1.112(1.083-1.236)$ & $<0.001$ & $1.100(1.080-1.250)$ \\
\hline High thrombus burden & 0.020 & $1.423(1.062-1.854)$ & 0.030 & $1.600(1.470-2.760)$ \\
\hline Long target lesion & 0.027 & $1.269(1.156-1.405)$ & 0.019 & $1.948(1.908-1.990)$ \\
\hline
\end{tabular}

OR: odds ratio; CI: confidence interval. Variables entered univariate analysis were age, reperfusion time, SBP on admission, Killip classes, IABP use before PCI, type of occlusion, target lesion length, thrombus burden, initial TIMI flow, reference diameter, and method of reperfusion. Killip classes, type of occlusion, reference diameter, and method of reperfusion were not included in multivariate analysis because they did not have a $P$ value $<0.05$ in univariate analysis. 
may improve myocardial reperfusion by alleviating the adverse effects of organized thrombus. ${ }^{[26]}$ In addition, noreflow may occur even in patients with AMI who have a low thrombus burden and a long reperfusion time. In that case, the material potential to embolize is small, extended ischemia can disrupt the microvascular bed, and the degree of this disruption is known to be a key factor in the pathogenesis. ${ }^{[1]]}$

Patients who had a low $(\leq 1)$ TIMI flow in the IRA prior to PCI had a higher rate of no-reflow than those with good $(\geq 2)$ TIMI flow on baseline angiography. De Luca et $\mathrm{al}^{[12]}$ found that pre-PCI good TIMI flow was strongly related to post-procedural TIMI 3 flow, myocardial blush grade 2-3 and lower enzymatic infarct size. Good patency of the IRA prior to PCI suggests a lower thrombus burden, spontaneous endogenous lysis of the thrombus, resolution of vasospasm and smaller infarct size. Thus, treatment of AMI should focus on the patency of the IRA and achieving a sustained antegrade blood flow as soon as possible. Although primary PCI is superior to thrombolytic therapy in achieving a TIMI 3 flow, its main limitation is the time delay on transferring patients or social problem. If a delayed primary PCI is expected, platelet glycoprotein IIb/IIIa receptor antagonists or low dose thrombolytic drugs in the early phase of AMI should be administered to restore early IRA patency and increase the chances of achieving a post-procedural TIMI 3 flow. ${ }^{[13]}$

Lee et $\mathrm{al}^{[14]}$ reported that a systolic $\mathrm{BP}(\mathrm{SBP})<120$ $\mathrm{mmHg}$ in patients with AMI was associated with a higher mortality than in those with SBP $>120 \mathrm{mmHg}$. A previous study showed that a low SBP $<120 \mathrm{mmHg}$ decreased coronary blood flow (CBF), collateral blood flow, and increased infact size. ${ }^{[15]}$ Their findings showed that low normal BP is associated with decreased CBF. Furthermore, the decreased $\mathrm{CBF}$ accelerates leukocyte accumulation, increases trapping leukocytes in capillaries, adhesion of leukocytes in venules, and noreflow. ${ }^{[16]}$

AMI patients with cardiogenic shock and Killip calss 3 on admission generally needed IABP supported priprimary $\mathrm{PCI}$, and these patients had a higher no-reflow rate after the procedure. Killip class $\geq 3$ on admission may be resulted from a larger infarction caused by severe damage to the microvascular bed as well as decreased coronary perfusion pressure. This explains why the patients using IABP had a higher no-reflow rate. A study ${ }^{[17]}$ demonstrated that cardiac cells in the no-reflow area were swollen and that the capillary endothelium was damaged and exhibited regional swelling with large intraluminal protrusions. Thus we consider that cellular edema and cell contracture compressing the capillaries may contribute to microvascular compression. A higher rate of distal embolization was found in patients with advanced Killip class, which may partially explain no-reflow in these patients. ${ }^{[18]}$

Acute coronary syndromes result from plaque rupture or fissuring with superimposed thrombus formation. Microvascular embolization of plaque material and thrombus can be seen spontaneously or iatrogenically during PCI. ${ }^{[19]}$ Claeys et a ${ }^{[20]}$ reported if the distal embolism of thrombus plugging the capillary lumen was $>50 \%$, decreased irreversible myocardial perfusion developed. The diameter of thrombi related to microvascular malfunction did not exceed $200 \mu \mathrm{m}$. Apart from distal embolism, thrombus can lead to impairment of autoregulation and be accompanied by local release of vasoconstrictors and cardiac sympathetic reflexes with microvascular dysfunction. These changes are related to angiographic no-reflow. Watanabe et $\mathrm{al}^{[21]}$ investigated preinterventional intravascular ultrasound (IVUS) findings, and they suggested a possible relationship between lipid-rich plaque and no-reflow. Tanaka et al ${ }^{[22]}$ used IVUS to examine plaque burden and identified a higher lipid content in the plaque inner core and width of the external elastic membrane as independent markers for the no-reflow phenomenon. Our study demonstrates that large lesioned vessels, especially those with the IRA diameter above $4 \mathrm{~mm}$, increased the occurrence of noreflow. Patients with a lesion larger than $20 \mathrm{~mm}$ were more likely to develop no-reflow after primary PCI than those with a lesion larger than $<20 \mathrm{~mm}$. Large vessels are able to contain large amounts of plaque lipid or thrombus. The larger the lesioned vessels, the slower the flow velocity. The longer the target lesion, the larger amount of thrombus and plaque burden. This would explain the high risk for slow/no-reflow observed in these patients after primary PCI. ${ }^{[23,24]}$

In summary, the pathogenesis of no-reflow is complex and multifactorial. The possible mechanisms associated with no-reflow include swelling of myocardial cells compressing microvascular vessels, swelling of capillary endothelium with large intraluminal protrusions to plug the capillary lumen, contracture of myocardial cells compressing the capillaries, accumulation of neutrophils plugging in the microvasculature, embolization of capillaries caused by platelet aggregation, microthrombi, plaque lipid fragment, microvascular bed disruption, capillary spasm and dysfunction, and reperfusion injury. Patients 
with advanced age, delayed reperfusion, low SBP on admission, IABP use before PCI, low TIMI flow and/ or high thrombus burden on baseline angiography and long target lesions are at an increased risk for no-reflow development. Pharmacologic agents i.e., adenosine, verapamil, platelet glycoprotein IIa/IIIbantagonists, nitroglycerin, sodium nitroprusside have favorable effects on microvasculature, and they may be of value for no-reflow development. In patients, the use of a distal protection device or aspiration thrombectomy has favorable effects. In our study, we achieved TIMI 3 flow in the IRA after predilation, yet the same patients developed no-reflow after stent implantation. So, it is important to avoid or minimize trauma to the vessel, avoid repetitive balloon dilatations and use the shortest stent if possible. In recent years, it has been shown that coronary stent implantation without predilation is feasible and can be performed safely in selected patients with AMI. ${ }^{[25]}$ Because most patients with AMI have a combination of these factors, combined treatment strategies should be preferred.

\section{Funding: None.}

Ethical approval: This study was approved by the Ethics Committee of Tongji University School of Medicine, Shanghai 200120, China.

Conflicts of interest: The authors declare that there is no conflict of interest.

Contributors: Zhou H proposed the study, analyzed the data and wrote the first draft. All authors contributed to the design and interpretation of the study and to further drafts.

\section{REFERENCES}

1 Gibson CM, de Lemos JA, Murphy SA, Marble SJ, McCabe $\mathrm{CH}$, Cannon $\mathrm{CP}$, et al. Combination therapy with abciximab reduces angiographically evident thrombus in acute myocardial infarction: a TIMI 14 substudy. Circulation 2001; 103: 25502554.

2 Kirma C, Izgi A, Dundar C, Tanalp AC, Oduncu V, Aung SM, et al. Clinical and prodedural predictors of no-reflow phenomenon after primary percutaneous coronary interventions: experience at a single center. Circ J 2008; 72: 716-721.

3 Reffelmann T, Kloner RA. The "no-reflow" phenomenon: basic science and clinical correlates. Heart 2002; 87: 162-168.

4 Wang CH, Chen YD, Yang XC, Wang LF, Wang HS, Sun ZJ, et al. A no-reflow prediction model in patients with ST-elevation acute myocardial infarction and primary drug-eluting stenting. Scand Cardiovasc J 2011; 45: 98-104

5 Lowe GD, Rumley A, Woodward M, Morrison CE, Philippou H, Lane DA, et al. Epidemiology of coagulation factors, inhibitors and activation markers: the Third Glasgow MONICA Survey. I. Illustrative reference ranges by age, sex and hormone use. Br J Haematol 1997; 97: 775-784.

6 Kirma C, Izgi A, Dundar C, Tanalp AC, Oduncu V, Aung SM, et al. Clinical and procedural predictors of no-reflow phenomenon after primary percutaneous coronary interventions: experience at a single center. Circ J 2008; 72: 716-721.

7 Hearse DJ, Bolli R. Reperfusion induced injury: manifestations, mechanisms, and clinical relevance. Cardiovasc Res 1992; 26: 101-108.

8 Ali A, Cox D, Dib N, Brodie B, Berman D, Gupta N, et al. Rheolytic thrombectomy with percutaneous coronary intervention for infarct size reduction in acute myocardial infarct size reduction in acute myocardial infarction:30-day results from a multicenter randomized study. J Am Coll Cardiol 2006; 48: 244-252.

9 Nagata Y, Usuda K, Uchiyama A, Uchikoshi M, Sekiguchi $\mathrm{Y}$, Kato $\mathrm{H}$, et al. Characteristics of the pathological images of coronary artery thrombi according to the infarct-related coronary artery in acute myocardial infarction. Circ J 2004; 68: 308-314.

10 Yip HK, Chen MC, Chang HW, Hang CL, Hsieh YK, Fang $\mathrm{CY}$, et al. Angiographic morphologic features of infarct-related arteries and timely reperfusion in acute myocardial infarction: predictors of slow-flow and no-flow. Chest 2002; 122: 13221332.

11 De Luca G, Suryapranata H, Zijlstra F, van 't Hof AW, Hoorntje JC, Gosselink AT, et al. Symptom-onset-to-balloon time and mortality in patients with acute myocardial infarction treated by primary angioplasty. J Am Coll Cardiol 2003; 42: 991-997.

12 De Luca G, Ernst N, Zijlstra F, van 't Hof AW, Hoorntje JC, Dambrink JH, et al. Preprocedural TIMI flow and mortality in patients with acute myocardial infarction treated by primary angioplasty. J Am Coll Cardiol 2004; 43: 1363-1367.

13 Zeymer U, Zahn R, Schiele R, Jansen W, Girth E, Gitt A, et al. Early epifibatide improves TIMI 3 patency before primary percutaneous coronary intervention for acute ST elevation myocardial infarction: Results of randomized integrilin in acute myocardial infarction (INTAMI) pilot trial. Eur Heart J 2005; 26: 1971-1977.

14 Lee KL, Woodlief LH, Topol EJ, Weaver WD, Betriu A, Col $\mathrm{J}$, et al. Predictors of 30-day mortality in the era of reperfusion for acute myocardial infarction: results from an international trialof 41021 patients. GUSTO-I Investigators. Circulation 1995; 91: 1659-1668.

15 Ishikura F, Miki A, Iwata A, Toshida T, Shakudo M, Asanuma $\mathrm{T}$, et al. Effect of systemic blood pressure in microcollateral circulation evaluated by real-time contrast echocardiography. $\mathrm{J}$ Am Soc Echocardiogr 2008; 21: 765-769.

16 Chen Y, Wang C, Yang X, Wang L, Sun Z, Liu H, et al. Independent no-reflow predictors in female patients with STelevation acute myocardial infarction treated with primary percutaneous coronary intervention. Heart Vessels 2012; 27: 243-249.

17 Movahed MR, Butman SM. The pathogenesis and treatment of no-reflow occurring during percutaneous coronary intervention. Cardiovasc Revasc Med 2008; 9: 56-61.

18 De Luca G, Gibson CM, Huber K, Zeymer U, Dudek D, Cutlip D, et al. Association between advanced Killip class ar presentation and impaired myocardial infarction treated with primary angioplasty and adjunctive glycoprotein IIb-IIIa 
inhibitors. Am Heart J 2009; 158: 416-421.

19 Skyschally A, Erbel R, Heusch G. Coronary microembolization. Circ J 2003; 67: 279-286.

20 Claeys MJ, Bosmans J, De Ceuninck M, Beunis A, Vergauwen $\mathrm{W}$, Vorlat A, et al. Effect of intracoronary adenosine infusion during coronary intervention on myocardial reperfusion injury in patients with acute myocardial infarction. Am J Cardiology 2004; 94: 9-13.

21 Watanabe T, Nanto S, Uematsu M, Ohara T, Morozumi T, Kotani J, et al. Prediction of no-reflow phenomenon after successful percutaneous coronary intervention in patients with acute myocardial infarction: intravascular ultrasound findings. Circ J 2003; 67: 667-671.

22 Tanaka A, Kawarabayashi T, Nishibori Y, Sano T, Nishida Y, Fukuda D, et al. No-reflow phenomenon and lesion morphology in patients with acute myocardial infarction. Circulation 2002; 105: 2148-2152.

23 Katayama T, Kubo N, Takagi Y, Funayama H, Ikeda N, Ishida $\mathrm{T}$, et al. Relation of atherothrombosis burden and volume detected by intravascular ultrasound to angiographic no-reflow phenomenon during stent implantation in patients with acute myocardial infarction. Am J Cardiol 2006; 97: 301-304.

24 Bae JH, Kwon TG, Hyun DW, Rihal CS, Lerman A. Predictors of slow flow during primary percutaneous coronary intervention:an intravascular ultrasound virtual histology study. Heart 2008; 94: 1559-1564.

25 Antoniucci D, Valenti R, Migliorini A, Moschi G, Bolognese L, Cerisano G, et al. Direct infarct artery stenting without predilation and no-reflow in patients with acute myocardial infarction. Am Heart J 2001; 142: 684-690.

26 Umeda H, Katoh T, Iwase M, Izawa H, Nagata K, Watanabe $\mathrm{K}$, et al. The distal protection during primary percutaneous coronary intervention alleviates the adverse effects of large thrombus burden on myocardial reperfusion. Circ J 2006; 70: $232-238$.

Received November 3, 2013 Accepted after revision April 16, 2014 